\title{
General quasi-overlap functions on lattices
}

\author{
Rui Paiva ${ }^{1}$ \\ IFCE, Maracanaú, CE \\ Benjamín Bedregal ${ }^{2}$ \\ DIMAp/UFRN, Natal, RN
}

\begin{abstract}
One recent work, Paiva et al. introduced the concept of quasi-overlap functions on bounded lattices and investigated some vital properties of them. In this paper, we continue consider this research topic and focus on a generalization, called general quasi-overlap functions, which measure the degree of overlapping of several classes in a given classification system and for any given object. We also provide a characterization, as well as some methods of constructing these functions.
\end{abstract}

Palavras-chave. Aggregation functions, General quasi-overlap functions, Lattices

\section{Introduction}

Aggregation functions has been widely studied in the fuzzy set theory. The importance of these functions is made apparent by their wide use, not only in theory of functional equations or measure and integration theory, but also in several applied fields. In [1], a mathematical model for medical diagnosis is given by equation $\mathcal{S} \otimes^{t} \mathcal{X}=\mathcal{T}$, where $\mathcal{S}$ and $\mathcal{T}$ are respectively the matricial forms of the fuzzy relations of symptoms and patients, with the product t-norm, then the diagnostic matrix is given by $\mathcal{D}=\mathcal{S}^{-1} \otimes_{g_{n}} \mathcal{T}$, where $g_{n}$ denotes the Goguen's implication. In [12] Sugeno integrals, for a given fuzzy measure, is studied under the viewpoint of aggregation and are used to determine the fuzzy expected valued applied to the accidents of traffic analysis, in the São Paulo city, Brazil. Moreover, a chapter is dedicated to fuzzy decision making in public health strategies based on fuzzy aggregation functions.

In this perspective, there are situations in which we need to measure the degree of overlapping of an object in a fuzzy classification system with more than two classes. In [17], to develop a classifier that tackles the problem of determining the risk of a patient of sufferingfrom a cardiovascular disease within the next 10 years, the authors used rules of the type:

$$
\text { Rule } R_{j} \text { : If } x_{p 1} \text { is } A_{j 1} \text { and } \ldots \text { and } x_{p n} \text { is } A_{j n} \text { then Class }=C_{j} \text { with } R W_{j} \text {, }
$$

where the inference procedure is an aggregation $A_{n}\left(\mu_{A_{j 1}}\left(x_{p 1}\right), \ldots, \mu_{A_{j n}}\left(x_{p n}\right)\right)$. In [7], the authors studied a special type of $n$-ary aggregation function on $[0,1]$, called general overlap functions, which measure the degree of overlapping (intersection for non-crisp sets) of $n$ different classes, for computing the matching degree in a classification problem. As one kind of binary general overlap functions, in [13] the authors extended the notion of BL-algebras, which are the algebraic counterpart of the a type of fuzzy logic modeled by Peter Hájek. This class of functions offers a promising field of research $[2,4,8,14,15]$. In [14], Paiva et al. introduced the concept of quasioverlap functions on bounded lattices and investigated some vital properties of them. In this

\footnotetext{
${ }^{1}$ rui.brasileiro@ifce.edu.br

2 bedregal@dimap.ufrn.br
} 
paper, we continue consider this research proposing a generalization of these functions, called general quasi-overlap functions, which are special aggregation functions on bounded lattices. From the applications point of view, the discussion of general quasi-overlap functions on bounded lattices will help us define new Choquet and Sugeno integral classes on bounded lattices that can provide us some potential applications in above fields.

Therefore, this paper is organized as follows: Section 2, in addition to establishing the notation used, we provides a review on lattices and aggregation functions on lattices. Moreover, to obtain a characterization theorem for general overlap functions, in the context of lattices, we recall the notion of quasi-groups, as well as some of their useful properties throughout this paper. Section 3 we formalize the notions of general overlap functions to the context of lattices. Moreover, a characterization and construction methods of general overlap functions are proposed. Finally, Section 4 gives some final remarks.

\section{Basic notions and terminology}

In this section, we recall some basic concepts and terminologies used throughout the paper.

\subsection{Aggregation functions on bounded lattices}

In this subsection, the reader is assumed to be familiar with some elementary notions of partial orders (or posets). For more details it is indicated $[3,6]$.

A poset $\left(X, \leq_{X}\right)$ where each pair of elements $x, y \in X$ has infimum and supremum, denoted respectively by $x \wedge y$ and $x \vee y$, is called lattice. If there are $0_{X}, 1_{X} \in X$ such that for all $x \in X$, $x \wedge 1_{X}=x$ and $x \vee 0_{X}=x$, then $\left(X, \leq_{X}\right)$ is called bounded lattice. Whenever the order $\leq_{X}$ is clear in the context, we will simply say that $X$ is a lattice. We now review concept of the Cartesian product of lattices. If $\left(X_{1}, \leq_{X_{1}}\right), \ldots,\left(X_{n}, \leq_{X_{n}}\right)$ are lattices and $\prod_{i=1}^{n} X_{i}=X_{1} \times \ldots \times X_{n}$ is the Cartesian product of the underlying sets, then also $\left(\prod_{i=1}^{n} X_{i}, \leq_{\text {comp }}\right)$ is a lattice, the so-called product lattice of $\left(X_{1}, \leq_{X_{1}}\right), \ldots,\left(X_{n}, \leq_{X_{n}}\right)$, where $\leq_{\text {comp }}$ is the componentwise partial order on the Cartesian product $\prod_{i=1}^{n} X_{i}$ given as follows: let $\vec{x}=\left(x_{1}, \ldots x_{n}\right)$ and $\vec{y}=\left(y_{1}, \ldots y_{n}\right)$ be two points of $\prod_{i=1}^{n} X_{i}$. Then, $\vec{x} \leq_{\text {comp }} \vec{y}$ if and only if $x_{i} \leq_{X_{i}} y_{i}$, for all $i=1, \ldots, n$. Let $X$ be a bounded lattice, and $n \in \mathbb{N}$ be fixed. An $n$-ary mapping $\psi: X^{n} \longrightarrow X$ is increasing if $\psi(\vec{x}) \leq_{X} \psi(\vec{y})$ whenever $\vec{x} \leq_{\text {comp }} \vec{y}$. If the orders $\leq_{X}$ and $\leq_{\text {comp }}$ are respectively replaced by the strict orders $<_{X}$ and $<_{\text {comp }}$, then one obtains a stronger requirement. A mapping with this property is called strictly increasing. On the other hand, if $\psi(\vec{y}) \leq_{X} \psi(\vec{x})$ whenever $\vec{x} \leq_{\text {comp }} \vec{y}$, then $\psi$ is a decreasing mapping. Similarly, strictly decreasing mappings are defined. Recent studies have focused on $n$-ary mappings on bounded lattices called aggregation functions $[5,11,14]$.

Definição 2.1 ([11]). Let $X$ be a bounded lattice. A mapping $F: X^{n} \longrightarrow X$ is called an aggregation function on $X$ whenever it is increasing and satisfies boundary conditions: $F\left(0_{X}, \ldots, 0_{X}\right)=0_{X}$ and $F\left(1_{X}, \ldots, 1_{X}\right)=1_{X}$.

We also remember that an $n$-ary aggregation function $F$ on a bounded lattice $X$ is called symmetric, if $F\left(x_{1}, \ldots, x_{i}, \ldots, x_{j} \ldots, x_{n}\right)=F\left(x_{1} \ldots, x_{j}, \ldots, x_{i} \ldots, x_{n}\right)$, for all $i, j \in\{1, \ldots, n\}$.

Important types of $n$-ary aggregation functions on lattice $X=[0,1]$ equipped with the usual ordering of real numbers are $n$-dimensional orvelap functions [10] and general overlap functions [7]. In Section 3, the notion of general overlap functions will be extended to the context of lattices. To 
obtain a characterization theorem for these functions, the next subsection will be dedicated to the algebraic structure used for this purpose.

\subsection{The algebra of quasigroups}

Let $(X, *)$ be a groupoid ${ }^{3}$ and let $y$ be any fixed element in $X$. The so-called translation maps $L_{y}$ and $R_{y}$ can be defined by $L_{y}(x)=y * x$ and $R_{y}(x)=x * y$ for all $x \in X$. It follows that $L_{y}: X \longrightarrow X$ and $R_{y}: X \longrightarrow X$ for each $y \in X$.

Definition 2.1 ([16]). The groupoid $(X, *)$ is called a quasigroup if the maps $L_{y}: X \longrightarrow X$ and $R_{y}: X \longrightarrow X$ are bijections for all $y \in X$.

Theorem 2.1 ([18]). A groupoid $(X, *)$ is a quasigroup if and only if for all ordered pairs $(y, z) \in$ $X^{2}$ there exist unique solutions $x, w \in X$ to the equations $x * y=z$ and $y * w=z$.

Let $(X, *)$ be a quasigroup and consider elements $x$ and $y$ of $X$. The element $x \backslash y$ of $X$ is defined as the unique solution $z$ of the equation $x * z=y$, i.e., $x *(x \backslash y)=y$. In other words, $L_{x}(z)=y$ if and only if $z=x \backslash y$. The element $x \backslash y$ may be read as " $x$ dividing $y$ " or " $x$ backslash $y$ ". The operation \on the set $X$ is known as left division (that is, $L_{x}^{-1}(y)=x \backslash y=z$ ) in the quasigroup $(X, *)$. The element $x / y$ of $X$ is defined as the unique solution $z$ of the equation $z * y=x$, i.e., $(x / y) * y=x$. In other words, $R_{y}(z)=x$ if and only if $x / y$. The element $x / y$ may be read as " $x$ divided by $y$ " or " $x$ slash $y$ ". The operation / on the set $X$ is known as right division (that is, $\left.R_{y}^{-1}(x)=x / y=z\right)$ in the quasigroup $(X, *)$. A groupoid $(X, *)$ is commutative means that $L_{y}(x)=R_{y}(x)$ for all $x \in X$.

Theorem 2.2 ([16]). If $(X, *)$ is a quasigroup which is associative, then $(X, *)$ necessarily has a unique identity element4 .

Therefore, with the exception of the empty quasigroup, each associative quasigroup is a group. A quasigroup is Abelian if it is commutative and associative, so is either empty or an Abelian group. Given an Abelian group $(X, *)$, we remember that for an element $a \in X$, any other $b \in X$ is called inverse of $a$, denoted by $b=a^{-1}$, when $a * b=b * a=e$.

Remark 2.1 ([19]). Let $(X, *)$ be a group, considered as a quasigroup. Then $x \backslash y=x^{-1} * y$ and $y / x=y * x^{-1}$. When a nonempty quasigroup is Abelian, since the operator $*$ is commutative, for any $x, y$ in $X$, its left division $x \backslash y$ and right division $y / x$ coincides and are denoted simply by $\frac{y}{x}$.

\section{General quasi-overlap functions}

In this section, the notion of general overlap functions will be extended to the context of lattices. Moreover, a characterization and construction methods of general overlap functions are proposed.

Definition 3.1. Let $X$ be a bounded lattice. The mapping $\mathcal{G O}: X^{n} \longrightarrow X$ is said to be a general quasi-overlap function on $X$, if the following conditions hold:

$(\mathcal{G O} 1) \mathcal{G O}$ is symmetric;

$(\mathcal{G O} 2) \mathcal{G O}\left(x_{1}, \ldots, x_{n}\right)=0_{X}$ if $x_{i}=0_{X}$, for some $i \in\{1, \ldots, n\}$;

$(\mathcal{G O} 3) \mathcal{G O}\left(x_{1}, \ldots, x_{n}\right)=1_{X}$ if $x_{i}=1_{X}$, for all $i \in\{1, \ldots, n\}$

\footnotetext{
${ }^{3}$ In the literature there are many nonequivalent definitions for groupoid. Here, a groupoid is an algebraic structure $(X, *)$ where $X$ is a non-empty set and $*$ is a binary function defined on $X$.

${ }^{4}$ An element $e$ is a left (right) identity element for quasigroup $(X, *)$ means that $L_{e}(x)=x\left(R_{e}(x)=x\right) \forall x \in X$.
} 
$(\mathcal{G O 4 )} \mathcal{G O}$ is increasing.

Example 3.1. (1) Let $X$ be a bounded lattice and $a \in X$. The map $\mathcal{G O}: X^{n} \longrightarrow X$ given by $\mathcal{G O}\left(x_{1}, \ldots, x_{n}\right)=\left\{\begin{array}{ll}\bigwedge_{i=1}^{n} x_{i} & \text { if } \bigvee_{i=1}^{n} x_{i} \leq a \\ \bigvee_{i=1}^{n} x_{i} & \text { otherwise }\end{array}\right.$ is a general quasi-overlap function on $X . A$ variant of this map given by $\mathcal{G O}\left(x_{1}, \ldots, x_{n}\right)= \begin{cases}\bigwedge_{i=1}^{n} x_{i} & \text { se } x_{i} \leq \text { a for each } i=1, \ldots, n \\ \bigvee_{i=1}^{n} x_{i} & \text { otherwise }\end{cases}$ is also a general quasi-overlap function on $X$.

(2) Let $X$ be a non-empty set and $(\wp(X), \subseteq)$ a lattice. The map $\mathcal{G O}: \wp(X)^{2} \longrightarrow \wp(X)$ given by $\mathcal{G O}\left(X_{1}, \ldots, X_{n}\right)=\left\{x_{i} \mid x_{i}=\bigcup_{i=1}^{n} X_{i}-\overline{\bigcap_{i=1}^{n} X_{i}}\right\}$ is a general quasi-overlap function on $\wp(X)$. A variant of this map given by $\mathcal{G O}\left(x_{1}, \ldots, x_{n}\right)=\left\{\begin{array}{ll}\emptyset & \text { if } \bigcap_{i=1}^{n} X_{i}=\emptyset \\ \bigcup_{i=1}^{n} X_{i} & \text { otherwise }\end{array}\right.$, is also a general quasi-overlap function on $\wp(X)$.

(3) Let $X$ be a non-empty set and $\mathcal{F}(X)$ the lattice of fuzzy sets on $X$, where the order considered is the inclusion of fuzzy sets. If $O:[0,1]^{n} \longrightarrow[0,1]$ is a general quasi-overlap function, then the map $\mathcal{G O}\left(A_{1}, \ldots, A_{n}\right)=\left\{\left(x, O\left(A_{1}(x), \ldots, A_{n}(x)\right)\right) \mid x \in X\right\}$ is a general quasi-overlap function on $\mathcal{F}(X)$.

Theorem 3.1. Let $\oplus$ and $\otimes$ be two increasing binary operations on a bounded lattice $X$, such that $\otimes$ distributes over $\oplus$. Suppose that $\left(X, \oplus, 0_{X}\right)$ is a quasigroup with identity element $0_{X},\left(X, \otimes, 1_{X}\right)$ is an Abelian quasigroup and $0_{X}$ is annihilator ${ }^{5}$ of $\otimes$. The n-ary function $\mathcal{G O}: X^{n} \longrightarrow X$ is a general quasi-overlap function if and only if

$$
\mathcal{G O}\left(x_{1}, \ldots, x_{n}\right)=\frac{f\left(x_{1}, \ldots, x_{n}\right)}{f\left(x_{1}, \ldots, x_{n}\right) \oplus g\left(x_{1}, \ldots, x_{n}\right)}
$$

for some $f, g: X^{n} \longrightarrow X$ such that

(i) $f$ and $g$ are symmetric;

(ii) $f\left(x_{1}, \ldots, x_{n}\right)=0$ if $x_{i}=0_{X}$, for some $i \in\{1, \ldots, n\}$;

(iii) $g\left(x_{1}, \ldots, x_{n}\right)=0$ if $x_{i}=1_{X}$, for all $i \in\{1, \ldots, n\}$;

(iv) $f$ is increasing and $g$ is decreasing;

(v) $f\left(x_{1}, \ldots, x_{n}\right) \oplus g\left(x_{1}, \ldots, x_{n}\right) \neq 0_{X}$.

Proof. $(\Rightarrow)$ Suppose that $\mathcal{G O}$ is a general quasi-overlap function and take $f(\vec{x})=\mathcal{G O}(\vec{x})$. By Theorem 2.1, for each $\vec{x} \in X^{n}$ and any $u \in X$, there is an unique $v \in X$ that satisfies the equation $\mathcal{G O}(\vec{x}) \oplus v=u(*)$. Define $g$ the function that associates the only $v \in X$ that satisfies $(*)$ with each $\vec{x} \in X^{n}$. In particular, if $x_{i}=1_{X}$, for all $i \in\{1, \ldots, n\}$, then $g\left(1_{X}, \ldots, 1_{X}\right)=1_{X} \backslash 1_{X}=0_{X}$ and $\mathcal{G O}(\vec{x}) \oplus g(\vec{x})=1_{X} \neq 0_{X}$. Thus, the function $\mathcal{G O}(\vec{x})=\frac{f(\vec{x})}{f(\vec{x}) \oplus g(\vec{x})}$ is well defined. Moreover,

\footnotetext{
${ }^{5}$ For all $x \in X, x \otimes 0_{X}=0_{X} \otimes x=0_{X}$.
} 
one easily verifies that conditions $(i),(i i i)$ and $(v)$ hold. For condition $(i i)$, if $x_{i}=0_{X}$, for some $i \in\{1, \ldots, n\}$, then $0_{X}=\frac{f(\vec{x})}{f(\vec{x}) \oplus g(\vec{x})}$ if and only if $0_{X} \otimes[f(\vec{x}) \oplus g(\vec{x})]=\left[\frac{f(\vec{x})}{f(\vec{x}) \oplus g(\vec{x})}\right] \otimes[f(\vec{x}) \oplus g(\vec{x})]$. As $0_{X}$ is annihilator of $\otimes$, the conclusion follows easily. For condition $(i v)$, as $f$ is increasing, $\vec{x} \leq_{\text {comp }} \vec{y}$ implies $f(\vec{x}) \leq_{X} f(\vec{y})$. Likewise, as $\otimes$ is commutative and increasing, it follows that

$$
\begin{aligned}
g(\vec{y}) & =f(\vec{y}) \backslash 1_{X} \\
& =f(\vec{y})^{-1} \otimes f(\vec{x})^{-1} \otimes f(\vec{x}) \\
& =[f(\vec{x}) \otimes f(\vec{y})]^{-1} \otimes f(\vec{x}) \\
& \leq X \\
= & {[f(\vec{x}) \otimes f(\vec{y})]^{-1} \otimes f(\vec{y}) } \\
& =f(\vec{x})^{-1} \otimes f(\vec{x})^{-1} \otimes f(\vec{y}) \\
& =f(\vec{x})^{-1} \otimes 1_{X} \\
& =f(\vec{x}) \backslash 1_{X} \\
& =
\end{aligned}
$$

$(\Leftarrow)$ Consider $f, g: X^{n} \longrightarrow X$ satisfying the conditions $(i)-(v)$. We show that the map of Equation (1) is a general quasi-overlap function on $X$. It is immediate that $\mathcal{G O}$ is symmetric $(\mathcal{G O} 1)$. Let us prove that the conditions $(\mathcal{G O} 2),(\mathcal{G O} 3)$ and $(\mathcal{G O} 4)$ hold. Let $\vec{x} \in X^{n}$ be such that $x_{i}=0_{X}$ for some $i \in\{1, \ldots n\}$. Due to conditions $(i i)$ and $(v)$, it holds that $f(\vec{x})=0_{X}$ and $f(\vec{x}) \oplus g(\vec{x}) \neq 0_{X}$. Consequently, $\mathcal{G O}(\vec{x})=0_{X}$. Similarly, let $\vec{x} \in X^{n}$ be such that $x_{i}=1_{X}$ for all $i \in\{1, \ldots n\}$. Due to conditions $(i i i)$ and $(v)$, it holds that $g(\vec{x})=0_{X}$ and $f(\vec{x}) \oplus g(\vec{x}) \neq 0_{X}$. Consequently, $\mathcal{G O}(\vec{x})=\frac{f(\vec{x})}{f(\vec{x})}=1_{X}$. Finally, let us see that $(\mathcal{G O} 4)$ also holds. Consider $\vec{x}, \vec{y} \in X^{n}$. Without loss of generality, suppose that $\vec{x} \leq_{\text {comp }} \vec{y}$. Due to condition $(i v)$, it holds that $f(\vec{x}) \leq_{X} f(\vec{y})$ and $g(\vec{y}) \leq_{X} g(\vec{x})$. Similarly, we find that $f(\vec{x}) \otimes g(\vec{y}) \leq_{X} f(\vec{y}) \otimes g(\vec{x})$. Since $\oplus$ also is increasing, it follows that $[f(\vec{x}) \otimes f(\vec{y})] \oplus[f(\vec{x}) \otimes g(\vec{y})] \leq_{X}[f(\vec{x}) \otimes f(\vec{y})] \oplus[f(\vec{y}) \otimes g(\vec{x})]$. As $\otimes$ distributes over $\oplus$, one has that $f(\vec{x}) \otimes(f(\vec{y}) \oplus g(\vec{y})) \leq_{X} f(\vec{y}) \otimes(f(\vec{x}) \oplus g(\vec{x}))$. Therefore, as $(X, \otimes)$ is an Abelian quasigroup, $\mathcal{G O}(\vec{x})=\frac{f(\vec{x})}{f(\vec{x}) \oplus g(\vec{x})} \leq_{X} \frac{f(\vec{y})}{f(\vec{y}) \oplus g(\vec{y})}=\mathcal{G O}(\vec{y})$.

Theorem 3.2. Let $X$ be a totally ordered bounded lattice and $F: X^{n} \longrightarrow X$ a symmetric aggregation function. If $F\left(x_{1}, \ldots, x_{n}\right) \leq_{X} \min \left(x_{1}, \ldots, x_{n}\right)$, then $F$ is a general quasi-overlap function.

Proof. Since $F$ is symmetric, $(\mathcal{G O} 1)$ is satisfied. Since $F$ is an aggregation function, by boundary condition $(\mathcal{G O} 3)$ is satisfied. Also, it follows that $F$ is increasing and so $(\mathcal{G O} 4)$ is satisfied. Moreover, if $x_{i}=0_{X}$, for some $i \in\{1, \ldots, n\}$, then $F\left(x_{1}, \ldots, x_{n}\right) \leq_{X} \min \left(x_{1}, \ldots, x_{n}\right)=0_{X}$. Thus $F\left(x_{1}, \ldots, x_{n}\right)=0_{X}$, hence $(\mathcal{G O} 2)$ is satisfied. Thus, $F$ is a general overlap function on $X$.

Theorem 3.3. Let $X$ be a totally ordered bounded lattice, $\psi: X \longrightarrow X$ be an increasing function satisfying $\psi\left(0_{X}\right)=0_{X}$ and $\psi\left(1_{X}\right)=1_{X}$. Moreover, let $F: X^{n} \longrightarrow X$ be a symmetric aggregation function. If $\psi\left(F\left(1_{X}, \ldots, 1_{X}, t, 1_{X}, \ldots, 1_{X}\right)\right) \leq_{X} t$, for all $t \in X$, and at any position, then $\mathcal{G O}=$ $\psi \circ F$ is a general quasi-overlap function.

Proof. For any fixed position $i$, and any $\vec{x}=\left(x_{1}, \ldots, x_{n}\right)$ we have $\mathcal{G O}(\vec{x}) \leq_{X} \max _{x_{j} \in X, j \neq i} \mathcal{G O}(\vec{x})=$ $\mathcal{G O}\left(1_{X}, \ldots, 1_{X}, x_{i}, 1_{X}, \ldots, 1_{X}\right) \leq_{X} x_{i}$. This holds for every $i$, thus $\mathcal{G O}(\vec{x}) \leq_{X} \min (\vec{x})$. By applying Theorem 3.2 we complete the proof.

Theorem 3.4. Let $X$ be a bounded lattice and $\rho_{1}, \ldots, \rho_{n}, \psi: X \longrightarrow X$ strictly increasing bijections. For any general quasi-overlap function $\mathcal{G O}$, the mapping

$$
\widetilde{\mathcal{G O}}\left(x_{1}, \ldots, x_{n}\right)=\psi\left(\mathcal{G O}\left(\rho_{1}\left(x_{1}\right), \ldots, \rho_{n}\left(x_{n}\right)\right)\right)
$$


is a general quasi-overlap function.

Proof. The property $(\mathcal{G O} 1): \widetilde{\mathcal{G O}}$ is symmetric, since $\mathcal{G O}$ is symmetric. The property $(\mathcal{G O} 4)$, it follows from the fact that the mappings $\rho_{1}, \ldots, \rho_{n}, \psi$ are strictly increasing and $\mathcal{G O}$ is increasing. As for the properties $(\mathcal{G O} 2)$ and $(\mathcal{G O} 3)$, they follow from the fact that for all strictly increasing bijection $\varphi: X \longrightarrow X$ one has that $\varphi(x)=0_{X}$ if and only if $x=0_{X}$ and $\varphi(x)=1_{X}$ if and only if $x=1_{X}$. Indeed, since $\varphi$ is surjective, there are $u, w \in X$ such that $\varphi(u)=0_{X}$ and $\varphi(w)=1_{X}$. Obviously, if $0_{X}<_{X} u$ and $w<_{X} 1_{X}$, as $\varphi$ is strictly increasing, we have the contradictions $\varphi\left(0_{X}\right)<_{X} \varphi(u)=0_{X}$ and $\varphi(w)=1_{X}<_{X} \varphi\left(1_{X}\right)$. Thus $u=0_{X}$ and $w=1_{X}$. Moreover, if $\varphi\left(0_{X}\right)=m$, where $0_{X}<m$, then since $\varphi$ is bijection, there is $n \in X$ such that $0_{X}<_{X} n$ and $\varphi(n)=0_{X}$. Hence, $\varphi(n)<_{X} \varphi\left(0_{X}\right)$, which contradicts the fact that $\varphi$ is strictly increasing. Similarly, if $\varphi\left(1_{X}\right)=s$, where $s<1_{X}$, then since $\varphi$ is bijection, there is $r<_{X} 1_{X}$ such that $\varphi(r)=1_{X}$. Hence, $\varphi\left(1_{X}\right)<_{X} \varphi(r)$, obviously another contradiction. Hence, since $\mathcal{G O}$ is general quasi-overlap function, if $x_{i}=0_{X}$ for some $i \in\{1, \ldots, n\}$, it follows that $\rho_{i}\left(x_{i}\right)=0_{X}$, which implies that $\mathcal{G O}\left(\rho_{1}\left(x_{1}\right), \ldots, \rho_{n}\left(x_{n}\right)\right)=0_{X}$ and so $\psi\left(\mathcal{G O}\left(\rho_{1}\left(x_{1}\right), \ldots, \rho_{n}\left(x_{n}\right)\right)\right)=0_{X}$. Therefore, $\widetilde{\mathcal{G O}}\left(x_{1}, \ldots, x_{n}\right)=0_{X}$. On the other hand, if $x_{i}=1_{X}$ for all $i \in\{1, \ldots, n\}$, it follows that $\rho_{i}\left(x_{i}\right)=$ $1_{X}$, which implies that $\mathcal{G O}\left(\rho_{1}\left(x_{1}\right), \ldots, \rho_{n}\left(x_{n}\right)\right)=1_{X}$. Then $\psi\left(\mathcal{G O}\left(\rho_{1}\left(x_{1}\right), \ldots, \rho_{n}\left(x_{n}\right)\right)\right)=1_{X}$, which implies that $\widetilde{\mathcal{G O}}\left(x_{1}, \ldots, x_{n}\right)=1_{X}$.

\section{Final remarks}

In this paper we have studied the concept of general quasi-overlap function on lattices. We have proved a characterization theorem and some construction methods for these functions. In [9], Dimuro et al. proposes some generalizations of the standard form of the Choquet Integral and among these generalizations, one uses a particular type of aggregation functions, called overlap functions, which are a particular class of quasi-overlap functions. In this perspective, for future research, we propose a generalization of the standard form of the Choquet integral, using general quasi-overlap functions on lattices, in order to obtain applications in many areas, such as in decision making and multi-criteria decision making or multi-criteria preferences, especially for the applications of discrete Choquet integrals in fuzzy rule-based classification systems and ensembles of classifiers.

\section{References}

[1] De Barros, L. C., Bassanezi, R. and Lodwick, W. A First Course in Fuzzy Logic, Fuzzy Dynamical Systems and Biomathematics Theory and Applications. In Studies in Fuzziness and Soft Computing. Springer, 2017. ISSN 1434-9922.

[2] Bedregal, B., Bustince, H., Palmeira, E., Dimuro, G. and Fernandez, J. Generalized interval-valued OWA operators with interval weights derived from interval-valued overlap functions, International Journal of Approximate Reasoning, 90:1-16, 2017. DOI: 10.1016/j.ijar.2017.07.001.

[3] Birkhoff, G. Lattice Theory, 3rd edition. American Mathematical Society, Providence, 1967.

[4] Da Cruz, A. T., Dimuro, G. P., Bedregal, B., Sanz, J. A., Pereira, S. and Bustince, H. General interval-valued overlap functions and interval-valued overlap indices, Information Sciences, 527:27-50, 2020. DOI: 10.1016/j.ins.2020.03.091. 
[5] Dan, Y., Hu, B. and Qiao, J. General L-fuzzy aggregation functions based on complete residuated lattices, Soft Comput, 24:3087-3112, 2020. DOI: 10.1007/s00500-019-04642-8.

[6] Davey, B. A. and Priestley, H. A. Introduction to Lattices and Order, 2rd edition. Cambridge University Press, Cambridge, 2002.

[7] De Miguel, L., Gómez, D., Rodríguez, J. T., Montero, J., Bustince, H., Dimuro, G. P. and Sanz, J. A. General overlap functions, Fuzzy Sets and Systems, 372:81-96, 2018. DOI: 10.1016/j.fss.2018.08.003.

[8] Dimuro, G. P., Bedregal, B., Bustince, H., Asiáin, M. J. and Mesiar, R. On additive generators of overlap functions, Fuzzy Sets and Systems, 287:76-96, 2016. DOI: 10.1016/j.fss.2015.02.008.

[9] Dimuro, G. P., Fernández, J., Bedregal, B., Mesiar, R., Sanz, J. A., Lucca, G. and Bustince, H. The state-of-art of the generalizations of the Choquet integral: From aggregation and pre-aggregation to ordered directionally monotone functions, Information Fusion, 57:27-43, 2020. DOI: $10.1016 /$ j.inffus.2019.10.005.

[10] Gómez, D., Rodríguez, J. T., Montero, J., Bustince, H. and Barrenechea, E. n-dimensional overlap functions, Fuzzy Sets and Systems, 287:57 - 75, 2016. DOI: 10.1016/j.fss.2014.11.023.

[11] Karaçal, F. and Mesiar, R. Aggregation functions on bounded lattices, International Journal of General Systems, 46:37-51, 2017. DOI: 10.1080/03081079.2017.1291634.

[12] Massad, E., Ortega, N., de Barros, L. and Struchiner, C. Fuzzy Logic in Action: Applications in Epidemiology and Beyond. In Studies in Fuzziness and Soft Computing. Springer Berlin Heidelberg, 2009. ISSN: 1434-9922.

[13] Paiva, R., Santiago, R., Bedregal, B. and Rivieccio, U. Inflationary BL-algebras obtained from 2-dimensional general overlap functions, Fuzzy Sets and Systems, 418:64-83, 2021. DOI: 10.1016/j.fss.2020.12.018.

[14] Paiva, R., Santiago, R., Bedregal, B. and Palmeira, E. Lattice-valued overlap and quasioverlap functions, Information Sciences, 562:180-199, 2021. DOI: 10.1016/j.ins.2021.02.010.

[15] Paiva, R., Bedregal, B., Santiago, R. and Vieira, T. Residuated implications derived from quasi-overlap functions on lattices, International Journal of Approximate Reasoning, 134:95110, 2021. DOI: 10.1016/j.ijar.2021.04.008.

[16] Pflugfelder, H. Quasigroups and loops: introduction. In Sigma series in pure mathematics. Heldermann Verlag, 1990. ISSN: 0936-8272.

[17] Sanz, J. A., Galar, M., Jurio, A., Brugos, A., Pagola, M. and Bustince, H. Medical diagnosis of cardiovascular diseases using an interval-valued fuzzy rule-based classification system, Applied Soft Computing, 20:103-111, 2014. DOI: 10.1016/j.asoc.2013.11.009.

[18] Shcherbacov, V. Elements of Quasigroup Theory and Applications. In Chapman and Hall/CRC Monographs and Research Notes in Mathematics Series. Taylor \& Francis, 2017. ISSN: 2372-9309.

[19] Smith, J. Introduction to Abstract Algebra (Textbooks in Mathematics), 1rd edition. Taylor \& Francis, Ames 2008. 